DFTT 08/2005

LAPTH-1104/05

\title{
Antiproton fluxes from light neutralinos
}

\author{
A. Bottino, F. Donato, and N. Fornengo \\ Dipartimento di Fisica Teorica, Università di Torino \\ Istituto Nazionale di Fisica Nucleare, via P. Giuria 1, I-10125 Torino, Italy \\ P. Salati \\ Laboratoire d'Annecy-le-Vieux de Physique Théorique LAPTH \\ $C N R S-S P M$ and Université de Savoie \\ 9, Chemin de Bellevue, \\ B.P.110 74941 Annecy-le-Vieux, France
}

(Dated: September 22, 2018)

\begin{abstract}
We analyze how the measurements of the low-energy spectrum of cosmic antiprotons can provide information on relic neutralinos. The analysis is focused on the light neutralinos which emerge in supersymmetric schemes where gaugino-mass unification is not assumed. We determine which ranges of the astrophysical parameters already imply stringent constraints on the supersymmetric configurations and those ranges which make the antiproton flux sensitive to the primary component generated by the neutralino self-annihilation. Our results are derived from some general properties of the antiproton flux proved to be valid for a generic cold dark matter candidate.
\end{abstract}

PACS numbers: 95.35.+d,98.35.Gi,98.35.Pr,96.40.-z,98.70.Sa,11.30.Pb,12.60.Jv,95.30.Cq

\section{INTRODUCTION}

In supersymmetric schemes, where gaugino-mass unification is not assumed, the lower bound on the neutralino mass is determined by the upper limit on the contribution of cold dark matter (CDM) to the cosmological density parameter, $\left(\Omega_{\mathrm{CDM}}\right)_{\max } h^{2}$. Using the value $\left(\Omega_{\mathrm{CDM}}\right)_{\max } h^{2}=0.13$, derived from results of Refs. 1, 2], one obtains the lower bound $m_{\chi} \gtrsim 7 \mathrm{GeV}$ [3, 4]. This is at variance with the more commonly employed lower limit of about $50 \mathrm{GeV}$, derived from the experimental (LEP) lower bound on the chargino mass. The rich phenomenology related to possible light neutralinos, with masses within the range $7 \mathrm{GeV} \leq m_{\chi} \leq 50 \mathrm{GeV}$, has been discussed in Refs. [3, 4, 5, 6].

In particular, in Ref. [6] it was scrutinized the capability of indirect measurements of WIMPs (Weakly Interacting Massive Particles) to detect light neutralinos and it was concluded that measurements of cosmic antiprotons represent the most promising mean of indirect exploration. The analysis of the antiproton signal was based on a study of the antiproton flux at one single representative value of the antiproton kinetic energy $\left(T_{\bar{p}}\right.$ $=0.23 \mathrm{GeV})$. It was proved that, for a wide range of the astrophysical parameters, the antiproton signal due to neutralino pair-annihilation is within the level of detectability at small values of $m_{\chi}$.

In the present paper we extend the previous investigation of the antiproton signal by analyzing the detailed features of the expected theoretical spectra, and discuss how our results can be employed to determine the pres- ence of an actual primary signal or, at least, to derive significant constraints on supersymmetric configurations at small $m_{\chi}$. Our analysis is carried out in the perspective of a significant breakthrough in the determination of some relevant astrophysical parameters and in view of a sizeable improvement in the measurement of the cosmic antiprotons spectrum, as expected in forthcoming experiments in space.

This paper is organized as follows. In Sect. II we analyze some properties of primary cosmic antiproton fluxes due to self-annihilation of a generic candidate of cold dark matter. These features are derived in the standard scheme usually employed to describe the decoupling of cold particles from the primordial plasma. An upper bound is obtained for the antiproton flux. Then, in Sect. III a full evaluation of the antiproton spectrum is derived in the case of relic neutralinos. Results and perspectives are presented in Sect. IV

\section{A FEW PROPERTIES OF THE SELF-ANNIHILATION CROSS-SECTION FOR A GENERIC WIMP}

Cosmic primary antiprotons can originate from the hadronization of quarks and gluons produced in WIMP self-annihilation processes (we consider here selfconjugate WIMPs) 7, 8, 9, 10, 11]. Once antiprotons are produced in the dark halo, they diffuse and propagate throughout the Galaxy. The propagated antiproton differential flux at a generic point of coordinates $r, z$ in the Galactic rest frame ( $r$ is the radial distance from the 
Galactic center in the Galactic plane and $z$ is the vertical coordinate) is

$$
\Phi_{\bar{p}}(r, z, E)=\frac{v_{\bar{p}}}{4 \pi} \Upsilon \frac{d N}{d T_{\bar{p}}} S_{\text {astro }}^{\bar{p}}(r, z, E),
$$

with

$$
\Upsilon=\frac{1}{2} \xi^{2} \frac{\left\langle\sigma_{\mathrm{ann}} v\right\rangle_{0}}{m_{\chi}^{2}}
$$

Notations are as follows: $v_{\bar{p}}$ is the antiproton velocity, $\left\langle\sigma_{\mathrm{ann}} v\right\rangle_{0}$ is the average, over the Galactic velocity distribution, of the WIMP annihilation cross-section multiplied by the relative velocity. $S_{\text {astro }}^{\bar{p}}$ is a function which takes into account all the effects of propagation in the Galaxy and includes $\rho^{2}(r, z), \rho(r, z)$ being the galactic dark matter distribution. Here we take:

$$
\rho(r, z)=\rho_{l} \frac{a^{2}+R_{\odot}^{2}}{a^{2}+r^{2}+z^{2}},
$$

where $\rho_{l}$ is the total local dark matter density set at the value of $0.3 \mathrm{GeV} / \mathrm{cm}^{3}, a$ is a core parameter, $a=3.5$ $\mathrm{kpc}$ and $R_{\odot}=8 \mathrm{kpc}$. In the following we do not include any clumpiness effect. $\xi$ represents the fractional local density $\rho_{\chi}$ of our generic WIMP as compared to $\rho_{l}$, i.e. $\xi=\rho_{\chi} / \rho_{l}$. By applying the usual rescaling procedure [12], one has $\xi=\min \left[1, \Omega_{\chi} h^{2} /\left(\Omega_{\mathrm{CDM}} h^{2}\right)_{\min }\right]$. From the analyses of Refs. [1, 2] one derives that at $2 \sigma$ level the cosmologically interesting region for cold dark matter is: $0.095 \leq \Omega_{\mathrm{CDM}} h^{2} \leq 0.13$ (in what follows this will be denoted as the WMAP range for CDM abundance). Thus, for $\left(\Omega_{\mathrm{CDM}} h^{2}\right)_{\min }$ we use here the value $\left(\Omega_{\mathrm{CDM}} h^{2}\right)_{\min }=0.095$.

$d N_{\bar{p}} / d T_{\bar{p}}$ is the differential antiproton spectrum per annihilation event:

$$
\frac{d N_{\bar{p}}}{d T_{\bar{p}}}=\sum_{F} B R(\chi \chi \rightarrow \bar{p}+X) \frac{d N_{\bar{p}}^{F}}{d T_{\bar{p}}},
$$

where $F$ denotes the different annihilation final states, $B R(\chi \chi \rightarrow \bar{p}+X)$ the branching ratios and $d N_{\bar{p}}^{F} / d T_{\bar{p}}$ stands for the antiproton energy spectra in the $F$ channel.

Throughout this paper we will be interested in the antiproton differential flux at Earth $\left(r=R_{\odot}, z=0\right)$ as a function of the antiproton kinetic energy; this flux will be simply denoted as $\Phi_{\bar{p}}\left(T_{\bar{p}}\right)$.

We turn now to a discussion about some general properties of the quantities entering the flux factor $\Upsilon$. To make the discussion more transparent, we consider a scenario where the standard expansion in $\mathrm{S}$ and $\mathrm{P}$ waves for the thermally averaged product of the annihilation cross- section times the relative velocity of the self-interacting particles

$$
<\sigma_{\mathrm{ann}} v>\simeq \tilde{a}+\tilde{b} \frac{1}{x},
$$

holds ( $x$ is defined as $x=m_{\chi} / T, T$ being the temperature).

For relic particles in the Galactic halo $x \sim 10^{6}$, then, usually, a good approximation is:

$$
<\sigma_{\mathrm{ann}} v>_{0} \simeq \tilde{a}
$$

We recall that $\left\langle\sigma_{\text {ann }} v>\right.$ enters also in the relic abundance:

$$
\Omega_{\chi} h^{2}=\frac{x_{f}}{g_{\star}\left(x_{f}\right)^{1 / 2}} \frac{3.3 \times 10^{-38} \mathrm{~cm}^{2}}{\left\langle\widehat{\sigma_{\mathrm{ann}} v}\right\rangle},
$$

where $\left\langle\widetilde{\sigma_{\text {ann }} v}\right\rangle \equiv x_{f}\left\langle\sigma_{\text {ann }} v\right\rangle_{\text {int }},\left\langle\sigma_{\text {ann }} v\right\rangle_{\text {int }}$ being the integral of $\left\langle\sigma_{\mathrm{ann}} v\right\rangle$ from the present temperature up to the freeze-out temperature $T_{f} ; x_{f}$ is defined as $x_{f} \equiv$ $m_{\chi} / T_{f}$ and $g_{\star}\left(x_{f}\right)$ denotes the relativistic degrees of freedom of the thermodynamic bath at $x_{f}$. Using the expansion of Eq. (5), one obtains: $\left\langle\widehat{\left.\sigma_{\text {ann }} v\right\rangle} \simeq \tilde{a}+1 /\left(2 x_{f}\right) \tilde{b}\right.$. Since $x_{f} \simeq 20$, also the $\mathrm{P}$-wave contribution $\tilde{b}$ has to be retained in this case. In the following, we however specifically assume that $\tilde{a} \geq 1 /\left(2 x_{f}\right)|\tilde{b}|$.

\section{A. Lower bound on $<\sigma_{\text {ann }} v>_{0}$}

The cosmological upper bound $\Omega_{\chi} h^{2} \leq\left(\Omega_{\mathrm{CDM}} h^{2}\right)_{\max }$ implies that $\left\langle\widehat{\sigma_{\text {ann }} v}\right\rangle$ is limited from below. For a cold relic with a mass in the range $10 \mathrm{GeV} \lesssim m_{\chi} \lesssim 1 \mathrm{TeV}$, one has $g_{\star}\left(x_{f} \simeq 20\right) \sim 90$, so that $x_{f} / g_{\star}\left(x_{f}\right)^{1 / 2} \sim 2.2$ (with variations of order $10 \%$ ). Then, from Eq. (7) $\left\langle\widehat{\left.\sigma_{\text {ann }} v\right\rangle} \gtrsim 7.3 \times 10^{-38} \mathrm{~cm}^{2} /\left(\Omega_{\mathrm{CDM}} h^{2}\right)_{\max }\right.$. Using the value $\left(\Omega_{\mathrm{CDM}} h^{2}\right)_{\max }=0.13$, we obtain $\left\langle\widetilde{\left.\sigma_{\text {ann }} v\right\rangle} \gtrsim 5.6 \times\right.$ $10^{-37} \mathrm{~cm}^{2}$. If $\tilde{a} \geq 1 /\left(2 x_{f}\right)|\tilde{b}|$, this implies

$$
<\sigma_{\text {ann }} v>_{0} \gtrsim 3 \times 10^{-37} \mathrm{~cm}^{2} .
$$

\section{B. Upper bound on $\xi^{2}<\sigma_{\text {ann }} v>_{0}$}

Often it turns out that $<\sigma_{\mathrm{ann}} v>_{0}$ may be orders of magnitude larger than the lower limit of Eq. (8). However, this fact does not automatically imply very large values for the antiproton flux, since the relevant quantity which enters in the antiproton flux is not simply $<\sigma_{\text {ann }} v>_{0}$, but instead $\xi^{2}<\sigma_{\text {ann }} v>_{0}$, through 
the factor $\Upsilon$. Indeed, the quantity $\xi^{2}<\sigma_{\text {ann }} v>_{0}$ coincides with $<\sigma_{\text {ann }} v>_{0}$ when $\Omega_{\chi} h^{2} \geq\left(\Omega_{\mathrm{CDM}} h^{2}\right)_{\min }$, but it is proportional to $<\sigma_{\text {ann }} v>_{0} /\left\langle{\widetilde{\sigma_{\text {ann }} v}}^{2}\right.$ when $\Omega_{\chi} h^{2}<\left(\Omega_{\mathrm{CDM}} h^{2}\right)_{\min }$; then it has a maximum at $\Omega_{\chi} h^{2}=$ $\left(\Omega_{\mathrm{CDM}} h^{2}\right)_{\min }$ 13, 14]. Let us call $\eta$ the set of parameters of the particle-physics model which describes our generic cold relic. The property, that we have just discussed, states that the maximum of the quantity $\xi^{2}<\sigma_{\text {ann }} v>_{0}$ :

$$
\left(\xi^{2}<\sigma_{\text {ann }} v>_{0}\right)_{\max }=<\sigma_{\text {ann }} v>\left._{0}\right|_{\eta=\eta^{\prime}}
$$

occurs when the model parameters $\eta$ have values $\eta^{\prime}$, such that $\left(\Omega_{\chi} h^{2}\right)_{\eta=\eta^{\prime}}=\left(\Omega_{\mathrm{CDM}} h^{2}\right)_{\min }$, that is (using Eq. (7)), when

$$
\left(\frac{g_{\star}\left(x_{f}\right)^{1 / 2}}{x_{f}}<\widetilde{\sigma_{\operatorname{ann}} v}>\right)_{\eta=\eta^{\prime}}=\frac{3.3 \times 10^{-38} \mathrm{~cm}^{2}}{\left(\Omega_{\chi} h^{2}\right)_{\min }} .
$$

Using the estimate already employed above, i.e. $x_{f} / g_{\star}\left(x_{f}\right)^{1 / 2} \sim 2.2$, from Eq. (10) one obtains

$$
\left(\left\langle\widehat{\sigma_{\mathrm{ann}} v}>\right)_{\eta=\eta^{\prime}}=\frac{7.3 \times 10^{-38} \mathrm{~cm}^{2}}{\left(\Omega_{\chi} h^{2}\right)_{\min }} \simeq 7.7 \times 10^{-37} \mathrm{~cm}^{2},\right.
$$

where, in the last step, the value $\left(\Omega_{\chi} h^{2}\right)_{\min }=0.095$ is used. Thus, within a factor of 2 , the maximum of $\xi^{2}<\sigma_{\mathrm{ann}} v>_{0}$ is stable for all sets of parameters $\eta$ which satisfy Eq. (11) and is given by

$$
\left(\xi^{2}<\sigma_{\text {ann }} v>_{0}\right)_{\max } \simeq 8 \times 10^{-37} \mathrm{~cm}^{2} .
$$

If we take the mass $m_{\chi}$ to be one of the model parameters, this limit is independent of $m_{\chi}$, provided the other parameters vary within ranges which allow solutions of Eq. (11).

As a consequence of the previous properties, we find that the maximum of the factor $\Upsilon$, as a function of $m_{\chi}$, is expected to decrease simply as $\Upsilon_{\max } \propto m_{\chi}^{-2}$.

We note that the above properties apply also to any other primary flux of cosmic particles due to WIMP selfinteractions in the halo, $e . g$. to the gamma-ray or positron flux.

\section{PRIMARY COSMIC ANTIPROTON FLUX FROM SELF-ANNIHILATION OF LIGHT NEUTRALINOS}

Now we finalize our previous considerations to the case of light relic neutralinos.

\section{A. The Supersymmetric Model}

The supersymmetric scheme employed here is an effective Minimal Supersymmetric extension of the Standard Model (MSSM) at the electroweak scale, where gaugino-mass unification is not assumed. This is defined in terms of a minimal number of parameters, only those necessary to shape the essentials of the theoretical structure of MSSM and of its particle content. The assumptions that we impose at the electroweak scale are: a) all squark soft-mass parameters are degenerate: $\left.m_{\tilde{q}_{i}} \equiv m_{\tilde{q}} ; \mathrm{b}\right)$ all slepton soft-mass parameters are degenerate: $m_{\tilde{l}_{i}} \equiv m_{\tilde{l}}$; c) all trilinear parameters vanish except those of the third family, which are defined in terms of a common dimensionless parameter $A: A_{\tilde{b}}=A_{\tilde{t}} \equiv A m_{\tilde{q}}$ and $A_{\tilde{\tau}} \equiv A m_{\tilde{l}}$. As a consequence, the supersymmetric parameter space consists of the following independent parameters: $M_{2}, \mu, \tan \beta, m_{A}, m_{\tilde{q}}, m_{\tilde{l}}, A$ and $R \equiv M_{1} / M_{2}$. In the previous list of parameters we have denoted by $\mu$ the Higgs mixing mass parameter, by $\tan \beta$ the ratio of the two Higgs v.e.v.'s, by $m_{A}$ the mass of the CP-odd neutral Higgs boson, and by $M_{1}, M_{2}$ the $\mathrm{U}(1), \mathrm{SU}(2)$ gaugino masses, respectively.

In the numerical random scanning of the supersymmetric parameter space we use the following ranges: $1 \leq \tan \beta \leq 50,100 \mathrm{GeV} \leq|\mu|, M_{2} \leq 1000 \mathrm{GeV}$, $100 \mathrm{GeV} \leq m_{\tilde{q}}, m_{\tilde{l}} \leq 1000 \mathrm{GeV}, \operatorname{sign}(\mu)=-1,1$, $90 \mathrm{GeV} \leq m_{A} \leq 1000 \mathrm{GeV},-3 \leq A \leq 3$, in addition to the above mentioned range $0.01 \leq R \leq 0.5$. We impose the experimental constraints: accelerators data on supersymmetric and Higgs boson searches, measurements of the $b \rightarrow s+\gamma$ decay and of the branching ratio $B_{s} \rightarrow \mu^{+} \mu^{-}$, and measurements of the muon anomalous magnetic moment $a_{\mu} \equiv\left(g_{\mu}-2\right) / 2$. For the ranges used for these observable and other details of the model we refer to Ref. [6].

\section{B. The supersymmetric flux factor $\Upsilon$}

Figs. 11 2] 3 give the scatter plots of the quantities $<\sigma_{\text {ann }} v>_{0}, \xi^{2}<\sigma_{\text {ann }} v>_{0}$ and $\Upsilon$ versus $m_{\chi}$, limited to supersymmetric configurations which satisfy the approximation $\tilde{a} \geq 1 /\left(2 x_{f}\right)|\tilde{b}|$ employed above. (Red) crosses denote configurations with a relic abundance in the cosmologically relevant range $0.095 \leq \Omega_{\chi} h^{2} \leq 0.13$, (blue) dots denote susy configurations where rescaling is effective (i.e. neutralinos form a subdominant species of relic particles). In Fig. 1 we notice the effect of the lower bound on $<\sigma_{\text {ann }} v>_{0}$, implied by the cosmological upper bound on $\Omega_{\mathrm{CDM}} h^{2}$ (see Eq. (8)). The rapidly rising of the scatter plot as $m_{\chi}$ reaches the value of 45 $\mathrm{GeV}$ is due to the self-annihilation process through the 


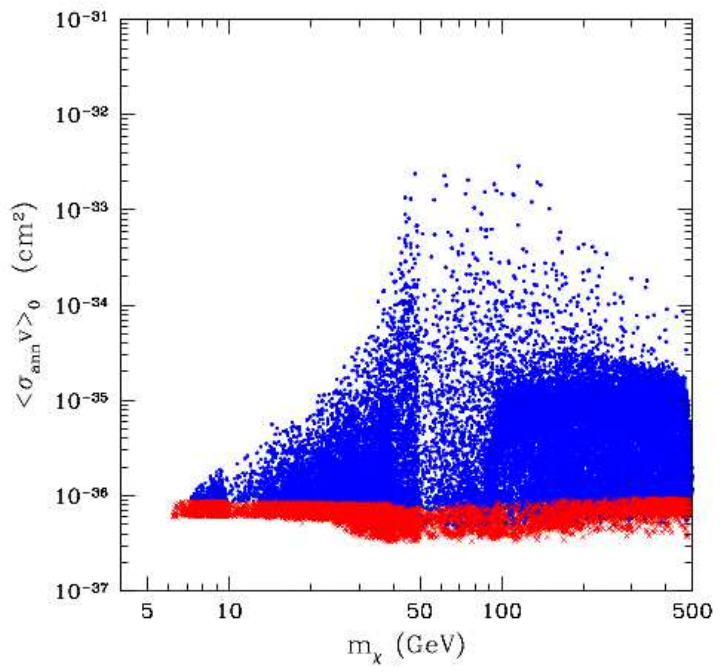

FIG. 1: Scatter plot of $\left\langle\sigma_{\mathrm{ann}} v>_{0}\right.$ vs $m_{\chi}$. Red crosses denote the supersymmetric configurations whose relic abundance is in the range $0.095 \leq \Omega_{\chi} h^{2} \leq 0.13$, while blue dots denote configurations with $\Omega_{\chi} h^{2} \leq 0.095$.

$Z$-boson exchange, superimposed to a similar enhancement extending to higher values of $m_{\chi}$, originating in the self-annihilation process with the exchange of the lightest CP-even neutral Higgs boson $h$. The upper frontier of the scatter plot at low values of $m_{\chi}$ is determined by the experimental lower bound on the mass of this Higgs boson.

Fig. 2 displays the upper bound on $\xi^{2}<\sigma_{\text {ann }} v>_{0}$, whose approximate estimate is given in Eq. (12).

Finally, we note that the upper frontier of the scatter plot for $\Upsilon$ in Fig. 3 clearly displays the simple behavior $\Upsilon_{\max } \propto m_{\chi}^{-2}$, as derived in Sect. [1B The lower part of the plot is composed by configurations with a large rescaling in the local density.

\section{The differential antiproton spectrum $d N_{\bar{p}} / d T_{\bar{p}}$}

To evaluate $d N_{\bar{p}} / d T_{\bar{p}}$, we follow the treatment of Ref. [6]. In case of neutralino masses below the thresholds for gauge-bosons, Higgs-bosons and $t$ quark production, antiprotons originate from the hadronization into $\bar{p}$ 's of the quark and gluon pairs produced in the neutralino selfannihilation. For light neutralinos $\left(m_{\chi} \leq 50 \mathrm{GeV}\right)$, which are mainly binos with a slight mixing with a higgsino component 4], the dominant final states are the ones into $b \bar{b}$ and into $\tau^{-} \tau^{+}$, the channel into $b \bar{b}$ being largely

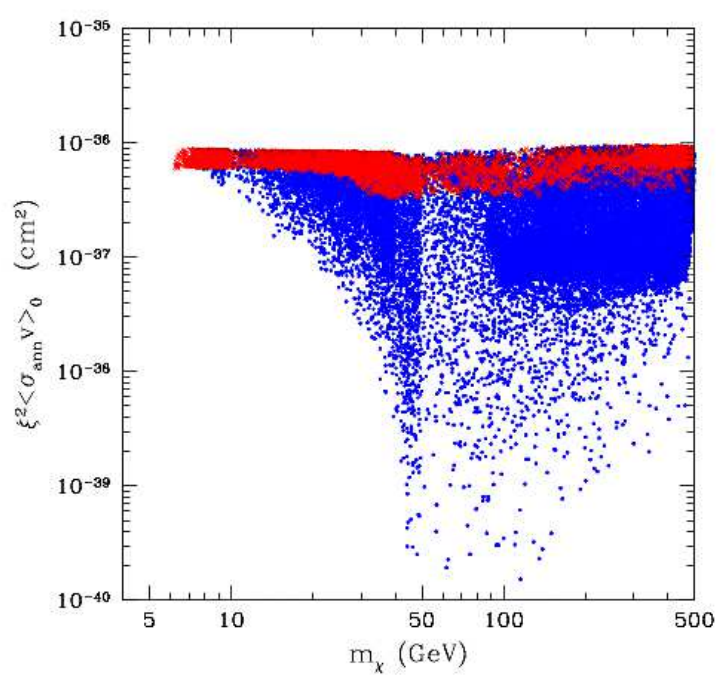

FIG. 2: Scatter plot of $\xi^{2}<\sigma_{\text {ann }} v>_{0}$ vs $m_{\chi}$. Notations as in Fig. 1

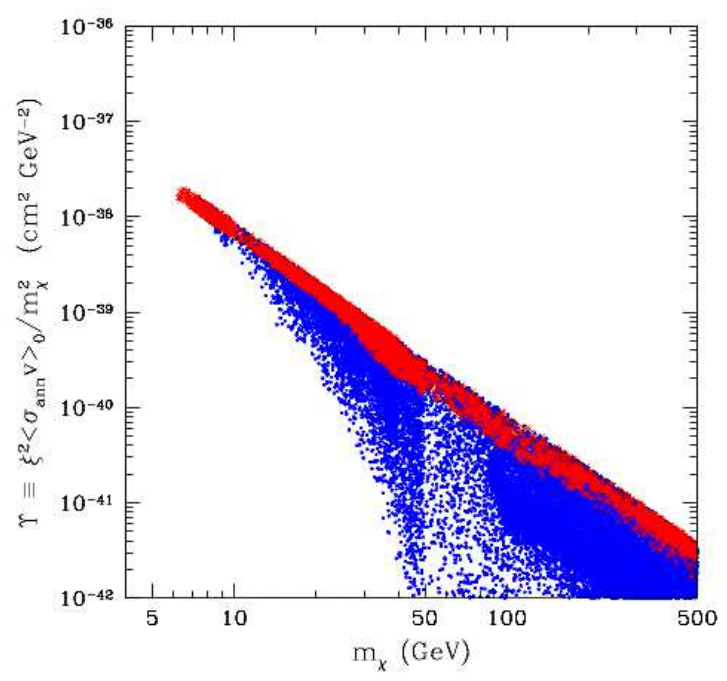

FIG. 3: Scatter plot of the quantity $\Upsilon$ vs $m_{\chi}$. Notations as in Fig. [

prominent for $m_{\chi} \lesssim 25 \mathrm{GeV}$. This property is displayed

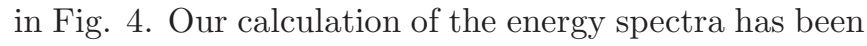
performed by using a Monte Carlo simulation with the PYTHIA package 15].

For neutralino masses which kinematically allow other final states (gauge-bosons, Higgs-bosons and $t \bar{t}$ pairs), 

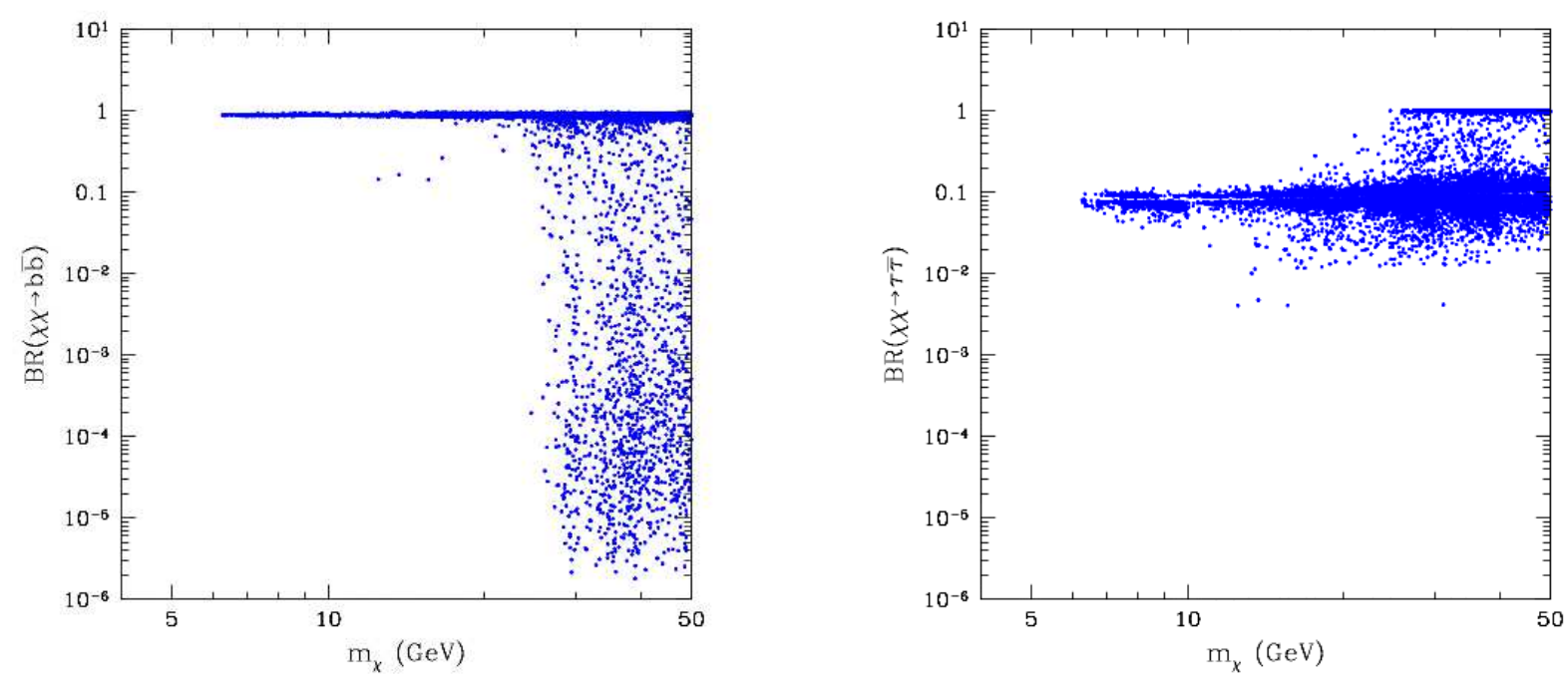

FIG. 4: Scatter plots of the branching ratios for the neutralino self-annihilation into $b \bar{b}$ (left panel) and $\tau^{+} \tau^{-}$vs $m_{\chi}$ (right panel).

the full decay chain down to the production of quarks and gluons has been evaluated analytically. The final antiproton spectrum is then calculated from the previous results by boosting the differential energy distribution to the rest frame of the annihilating neutralinos. Details of our procedure are given in Refs. 6, 11. A sample of results is shown in Fig. 5 where the spectra of antiprotons per annihilation event are shown for a sample of the neutralino masses and self-annihilation into $b \bar{b}$.

\section{Cosmic ray propagation in the Galaxy}

As they propagate throughout the Galaxy, charged cosmic rays mostly bounce on the spatial irregularities of its magnetic fields. That process is well described by mere diffusion with the energy dependent coefficient

$$
K=K_{0} \beta \mathcal{R}^{\delta}
$$

where $\mathcal{R}$ stands for the particle rigidity. The acceleration of primary species by supernovae driven shock waves as well as their subsequent interactions with the interstellar gas take place in a thin galactic disk that is sandwiched above and beneath by two large diffusion layers with thickness $L$. Because the magnetic field inhomogeneities - actually the Alfvén waves - move with speed $V_{A}$, cosmic rays undergo inside the disk some diffusive reacceleration that come into play with the ionization, Coulomb and adiabatic energy losses. Particles are fi-

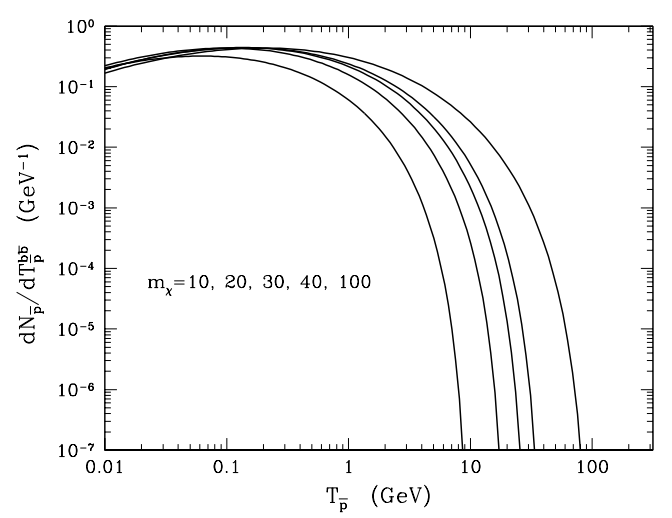

FIG. 5: The differential antiproton spectrum $d N_{\bar{p}} / d T_{\bar{p}}$ for annihilation in the $b \bar{b}$ channel, as a function of the antiproton kinetic energy $T_{\bar{p}}$. From left to right: $m_{\chi}=10,20,30,40$ and $100 \mathrm{GeV}$.

nally wiped away by a vertical convective wind whose velocity is $V_{c}$. The propagation of charged cosmic rays can be well accommodated in a cylindrical two-zone diffusion 
model. The particle radial abundances may be expanded as series of Bessel functions $J_{0}\left(\alpha_{i} r / R_{\text {gal }}\right)$ where $\alpha_{i}$ is the i-th zero of the function $J_{0}$ and where $R_{\text {gal }}=20 \mathrm{kpc}$ is the radius of the propagation region. For a complete description of the semi-analytic code on which the present analysis is based, we refer the reader to Ref. [16, 17, 18]. Suffice it to say that the five cosmic ray propagation parameters mentioned above - namely the diffusion coefficient normalization $K_{0}$ and index $\delta$, the confinement layers thickness $L$ and the velocities $V_{c}$ and $V_{A}$ - have been constrained by comparing the flux predictions for various cosmic ray species with observations. The most stringent observable is the boron to carbon ratio $\mathrm{B} / \mathrm{C}-\mathrm{a}$ typical secondary to primary relative abundance - whose analysis within our diffusion model has been detailed in Ref. 16, 17. The relevance of the latter has been further established by the compatibility of the $\mathrm{B} / \mathrm{C}$ results with several other observed cosmic ray abundances 17, 19, 20.

As a matter of fact, the conventional background for antiprotons is produced in the galactic disk by the spallation of proton and helium cosmic rays on the interstellar medium. A calculation of the flux at the Earth of that population of secondary antiprotons has been performed in Ref. 19] with the same propagation model as for the $\mathrm{B} / \mathrm{C}$ analysis. The secondary flux has been derived consistently by employing the propagation parameters that have been obtained from the full and systematic analysis of Ref. [16] on stable nuclei. The interstellar (IS) fluxes have been solar-modulated according to the force field approximation in order to obtain top-of-atmosphere (TOA) fluxes. Throughout this paper, we compare our results with data taken during minimal solar activity periods and we fix the modulation potential $\phi$ to $500 \mathrm{MV}$. It was found that the agreement between low-energy antiproton data and the estimate is excellent. Furthermore, the band within which the secondary flux lies is fairly restrained as shown in Fig. [6 In Ref. 19] it was actually inferred $\mathrm{a} \sim 20 \%$ astrophysical uncertainty - related to the propagation model - of the same order of magnitude as the theoretical error arising from nuclear physics. Taking adiabatic energy losses into account results - below a kinetic energy of $1 \mathrm{GeV}$ - into a small increase of our new secondary flux with respect to the previously published spectrum of Ref. [19].

Constraining cosmic ray propagation with $\mathrm{B} / \mathrm{C}$ provides a strong handle on any species that originates from the disk of the Milky Way and allows in particular the astrophysical uncertainties on secondary antiprotons to be mild as discussed above. At variance with that secondary component, primary antiprotons are produced by the annihilations of neutralinos spread all over the galactic halo. As extensively discussed in Ref. [1], the corresponding uncertainties span now two orders of magnitude, when

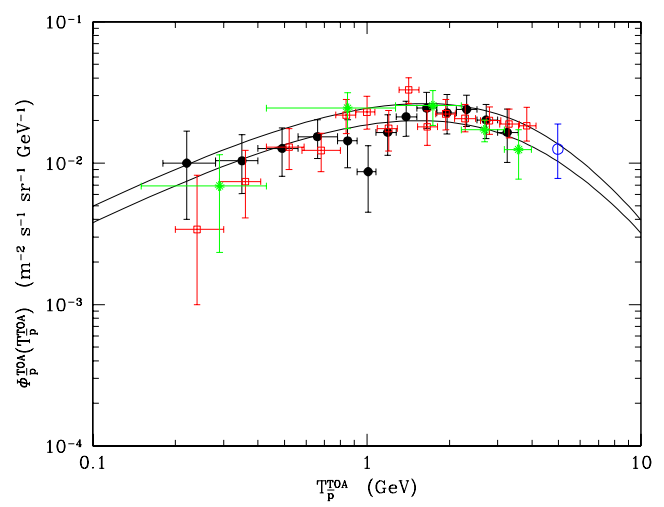

FIG. 6: The secondary antiproton flux lies within the uncertainty band - delineated by the two solid black lines - which corresponds to all the possible propagation schemes that are compatible with the B/C data. Experimental observations at solar minimum are featured, in good agreement with the theoretical estimate. Full circles: BESS 1995-97 [21]; open squares BESS 1998 [22]; stars: AMS 23]; empty circles: CAPRICE [24].

the propagation parameters are varied between the minimal and maximal configurations presented in Tab. [ Primary fluxes depend sensitively on the thickness of the

\begin{tabular}{|c|c|c|c|c|c|}
\hline case & $\delta$ & $\begin{array}{c}K_{0} \\
{\left[\mathrm{kpc}^{2} / \mathrm{Myr}\right]}\end{array}$ & $\begin{array}{c}L \\
{[\mathrm{kpc}]}\end{array}$ & $\begin{array}{c}V_{c} \\
{\left[\mathrm{~km} \mathrm{~s}^{-1}\right]}\end{array}$ & $\begin{array}{c}V_{A} \\
{\left[\mathrm{~km} \mathrm{~s}^{-1}\right]}\end{array}$ \\
\hline \hline $\max$ & 0.46 & 0.0765 & 15 & 5 & 117.6 \\
$\operatorname{med}$ & 0.70 & 0.0112 & 4 & 12 & 52.9 \\
$\min$ & 0.85 & 0.0016 & 1 & 13.5 & 22.4 \\
\hline
\end{tabular}

TABLE I: Astrophysical parameters compatible with B/C analysis and yielding the maximal, median and minimal primary antiproton flux.

confinement layers and also on the convective wind that wipes cosmic ray species away from the galactic disk. It is not surprising therefore if the largest primary antiproton yield corresponds to the combination $L=15 \mathrm{kpc}$ and $V_{c}=5 \mathrm{~km} \mathrm{~s}^{-1}$, whereas the smallest flux obtains when $L=1 \mathrm{kpc}$ and $V_{c}=13.5 \mathrm{~km} \mathrm{~s}^{-1}$. The Alfvénic velocity $V_{A}$ is strongly correlated with the normalization constant $K_{0}$ because the B/C ratio determines the diffusive reacceleration parameter $K_{E E} \propto V_{A}^{2} / K_{0}$ - see Ref. 11] with an accuracy of $\pm 15 \%$. The parameter $V_{A}$ will not be further discussed. Finally, we remind that - as obtained in Refs. [6, 11] - the specific form assumed for the dark matter distribution in the Galaxy is fairly irrelevant in the calculation of the propagated primary antiproton flux. In particular, for not too thick confinement lay- 
ers and strong convection winds, the cosmic ray diffusion range is small and solar circle abundances are blind to the center of the Milky Way and its putative neutralino cusp.

\section{RESULTS AND CONCLUSIONS}

Because our analysis is focused on light neutralinos $m_{\chi} \leq 40 \mathrm{GeV}$ - our main interest is in low-energy antiprotons. That is why we have selected the observations performed in the GeV region by Bess 1995-97 21], Bess 1998 [22] and AMS 1998 [23]. All these experiments operated at solar minimum for which $\phi=500 \mathrm{MV}$. The corresponding 32 data points are presented in Fig. 6] and are already very well explained by a pure secondary component. Actually, we have derived a $\chi^{2}$ of 33.6 in the case of the median configuration of Tab. I As discussed in Sec. IIID should the galactic cosmic ray propagation parameters be varied so as to keep the $\mathrm{B} / \mathrm{C}$ reduced $\chi^{2}$ below a generous value of 1.8 , the secondary antiproton flux would change by less than 20\% - see Ref. [19]. To put our discussion on a quantitative basis, we need to establish a criterion for deciding whether or not a specific supersymmetric configuration is excluded by the above mentioned antiproton data. To this purpose, we compute the primary flux which such a configuration yields, add it to the secondary component and derive the corresponding $\chi^{2}$. Notice that Caprice [24] has been disregarded in our calculation as it operated at a slightly higher energy than the range in which we are interested. Then we decide that a specific supersymmetric configuration is excluded if the corresponding $\chi^{2}$ exceeds a critical value of 60 . For 32 degrees of freedom, this translates into a disagreement at the $99.5 \%$ C.L. It is worth stressing that our exclusion criterion is purposely taken on the conservative side.

A final word of caution is in order before we proceed. The problem is complicated by the fact that the galactic cosmic ray propagation model is not unique. Actually, in the present analysis, we have varied the five propagation parameters presented in Sec. IIID and considered all the possible combinations that are in good agreement with stable nuclei [16]. We keep the conservative attitude of selecting propagation models as long as they lead to a reduced $\chi_{\text {red } \mathrm{B} / \mathrm{C}}^{2} \leq 1.8$ on the $\mathrm{B} / \mathrm{C}$ data.

To commence, we focus our investigation on the supersymmetric configurations which the present data on low-energy cosmic ray antiprotons constrain most. Those configurations are associated to large antiproton yields and correspond to high values of the effective annihilation cross-section $\xi^{2}<\sigma_{\text {ann }} v>_{0}$. We concentrate here on cases A) and B) for which the neutralino relic density is relevant to cosmology. Case A) corresponds to the largest antiproton signal and consequently to the strongest constraint as is clear in the panel a of Fig. 7] where the $\chi^{2}$ is plotted as a function of the diffusive halo thickness $L$. (Black) squares, (green) big circles, (red) smaller circles and (blue) dots correspond to a neutralino mass $m_{\chi}$ of $10,20,30$ and $40 \mathrm{GeV}$, respectively. The constellation of points of the same shape (and color) - which corresponds to a specific neutralino mass - is obtained by scanning the entire set of cosmic ray propagation models that are compatible with $\mathrm{B} / \mathrm{C}-$ as discussed above. The effective annihilation cross-section $\xi^{2}<\sigma_{\text {ann }} v>_{0}$ reaches here a maximal value of $10^{-36} \mathrm{~cm}^{2}$ as may be readily inferred from the scatter plot of Fig. 2 and its upper boundary. Case B) corresponds to the minimal value of the annihilation cross-section $\left\langle\sigma_{\mathrm{ann}} v>_{0}\right.$ that is yet compatible with WMAP. The neutralino relic abundance is set to the upper bound $\left(\Omega_{\mathrm{CDM}}\right)_{\max } h^{2}=0.13$. The corresponding scatter plot is presented in panel b of Fig. [7] The lower horizontal line at $\chi^{2}=33.6$ corresponds to the fit with the secondary antiproton component alone. The upper horizontal line indicates the discriminating $\chi^{2}$ value above which the fit to the low-energy antiproton data is no longer acceptable. The large spread exhibited by the scatter plots of Fig. 7 illustrates the strong sensitivity of the primary antiproton flux to the astrophysical parameters that account for cosmic ray propagation throughout the Milky Way and its halo. This is at variance with the stability of the secondary flux as already remarked above. As is clear from panels a and b, light neutralinos of cosmological interest - i.e. with a relic abundance in the WMAP range - are compatible with the present data on cosmic ray antiprotons only if the parameter $L$ is on the very low side of its physical interval. Should the diffusive halo thickness $L$ turn out to be larger than 2 $\mathrm{kpc}$ - a quite realistic assumption given the existence of electron synchrotron radiation far above the galactic disk - neutralinos lighter than $20 \mathrm{GeV}$ would be excluded. If we now assume that $L \geq 5 \mathrm{kpc}$, we readily conclude that neutralinos with sizeable relic abundance must be heavier than $40 \mathrm{GeV}$. In order to illustrate how our $\chi^{2}$ exclusion criterion translates on the observations, we have plotted in Fig. 8 the total antiproton spectrum - (red) solid curve - for a $20 \mathrm{GeV}$ neutralino with maximal relic abundance. The median configuration of Tab. \has been selected for the cosmic ray propagation parameters. It corresponds to the best fit to the $\mathrm{B} / \mathrm{C}$ data. The primary (blue longdashed curve) and secondary (blue dotted line) components add up to yield an antiproton signal well in excess of the observations. The corresponding $\chi^{2}$ reaches actually the unacceptable value of 200 .

The last panel of Fig. 7 features supersymmetric configurations for which the effective annihilation cross- 

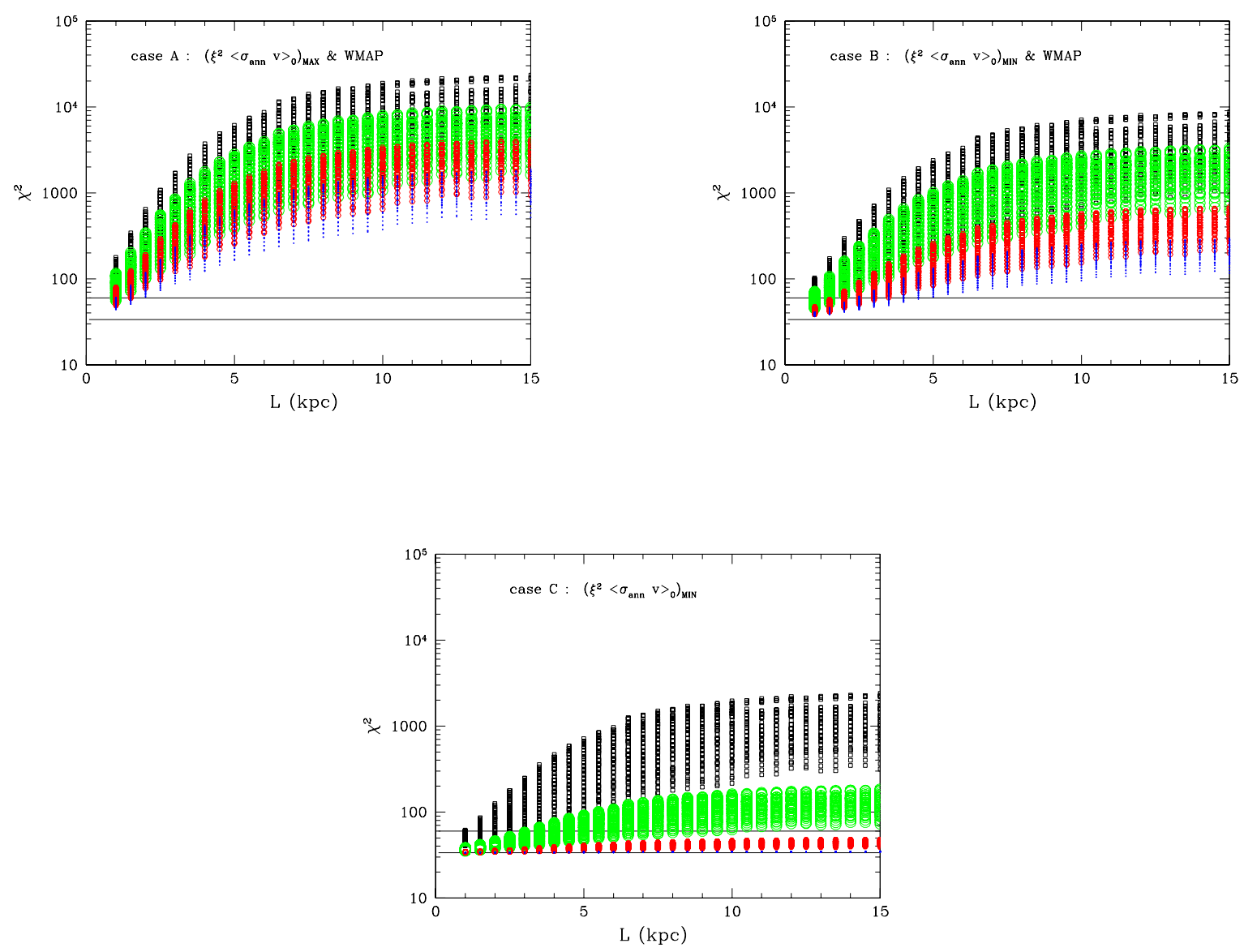

FIG. 7: The $\chi^{2}$ is featured as a function of the diffusive halo thickness $L$. Black squares, green big circles, red smaller circles and blue dots correspond to a neutralino mass $m_{\chi}$ of $10,20,30$ and $40 \mathrm{GeV}$, respectively. At fixed neutralino mass $m_{\chi}$, each point is associated to a specific combination of the galactic propagation parameters yielding a good fit for the B/C ratio. Panels a, b and c respectively correspond to cases A), B) and C) defined in the text. The upper horizontal line indicates the discriminating $\chi^{2}$ value above which the fit to the low-energy antiproton data is no longer acceptable. The lower horizontal line corresponds to the $\chi^{2}$ calculated with the secondary component alone for the median configuration of Tab. I

section $\xi^{2}<\sigma_{\text {ann }} v>_{0}$ is minimal and rescaling is maximal. The antiproton signal is at its weakest level and we do not expect low-energy antiproton data to be very constraining. Panel c of Fig. [7 indicates nevertheless that a $10 \mathrm{GeV}$ neutralino is excluded provided that the diffusive halo thickness $L$ exceeds $2.5 \mathrm{kpc}$. Should $L$ be larger than $10 \mathrm{kpc}$, the limit rises to $20 \mathrm{GeV}$. That panel shows also to what extent cosmologically subdominant neutralinos with a mass in the range $20 \mathrm{GeV} \lesssim m_{\chi} \lesssim 40 \mathrm{GeV}$ escape conflict with present data. A potential distortion is difficult to unravel from the antiproton spectrum given the available observations because error bars are too large. Actually, Fig. 9 and 10 feature respectively the primary antiproton flux of a 10 and $20 \mathrm{GeV}$ neutralino (blue long-dashed curves) in the case C) of maximal rescaling together with the conventional secondary component (blue dotted lines). The cosmic ray propa- gation configurations that have been extracted from the panel c of Fig. [7- a (black) square if the neutralino mass is $10 \mathrm{GeV}$ or a (green) big circle when $m_{\chi}$ is twice as large - lie on the upper horizontal line for which the fit to the low-energy antiproton data yields a $\chi^{2}$ value of 60 . Below an antiproton kinetic energy of $\sim 1 \mathrm{GeV}$, the global signal represented by the (red) solid curves is slightly shifted upwards with respect to the secondary spectrum whereas above $1 \mathrm{GeV}$, both spectra are identical. Richer samples of experimental data are a necessary condition for disentangling a supersymmetric signal from background secondary antiprotons. The spectrum of the latter suffers nevertheless from theoretical uncertainties cosmic ray propagation and nuclear cross-sections - that need to be further reduced in order to make antiproton measurements a useful probe for cosmologically subdominant neutralinos in the 10 to $30 \mathrm{GeV}$ range. 


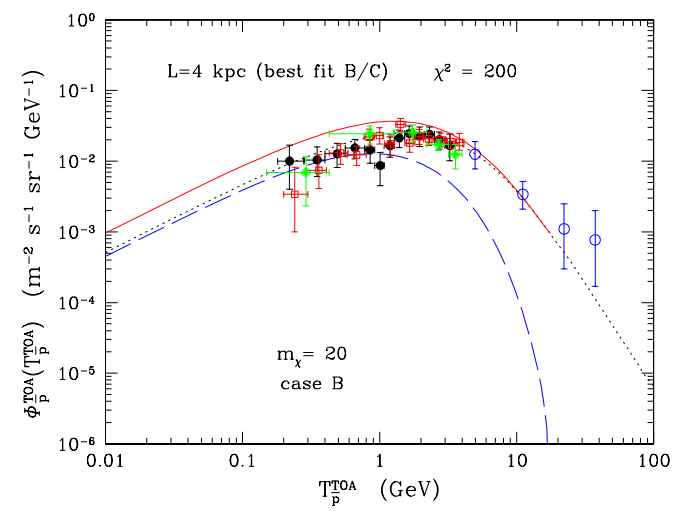

FIG. 8: The primary antiproton spectrum - blue long-dashed curve - has been computed for a $20 \mathrm{GeV}$ neutralino and corresponds to the case B) of a maximal relic abundance. The median configuration of Tab. \has been assumed. When added to the secondary component - blue dotted line - the primary signal results into a total antiproton flux - red solid spectrum - well above the data points. We actually infer a $\chi^{2}$ value of 200 to be compared to the exclusion limit of 60 . Symbols for experimental data as in Fig. [6]

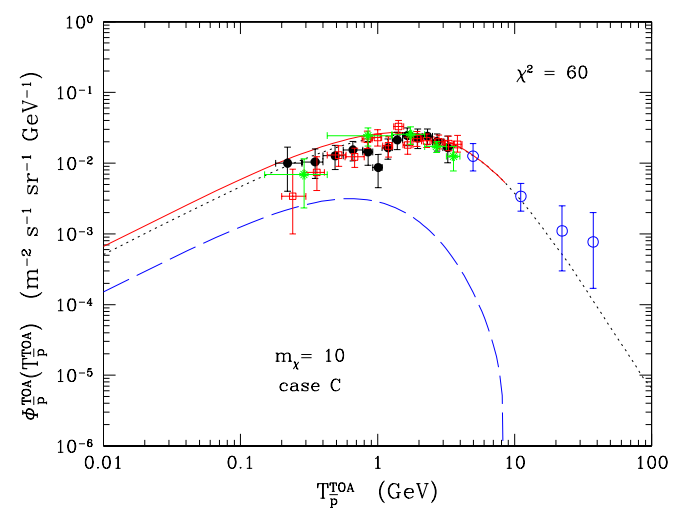

FIG. 9: The antiproton spectrum is featured together with its primary - blue long-dashed curve - and secondary - blue dotted line - components for a $10 \mathrm{GeV}$ neutralino. The supersymmetric configuration corresponds to maximal rescaling case C) - and the specific set of astrophysical parameters that has been extracted from panel c of Fig. [7 This case corresponds to $\chi^{2}=60$, and then is marginally acceptable. Symbols for experimental data as in Fig. [6]

It is very instructive to pursue the analysis of the maximal rescaling case C) in terms of other astrophysical parameters. The three scatter plots of Fig. [1] feature the same selection of astrophysical configurations drawn from Fig. (7- panel c - for which the fit to the antiproton data is acceptable with $\chi^{2} \leq 60$. Big (black) circles, smaller (green) circles and (red) dots correspond to a neutralino

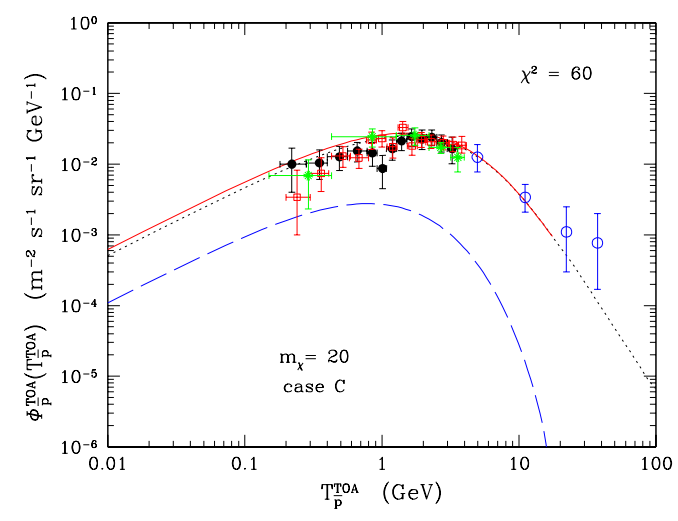

FIG. 10: Same as in Fig 9 with a $20 \mathrm{GeV}$ neutralino.

mass $m_{\chi}$ of 10,20 and $30 \mathrm{GeV}$ respectively. The smaller the latter, the more abundant the neutralinos at fixed mass density and the stronger the antiproton annihilation signal. This trend is clear in each of the panels of Fig. [1 It is even clearer in panel c. The relevant annihilation cross-section is in that case the minimal value of $\xi^{2}<\sigma_{\text {ann }} v>_{0}$ which increases rapidly with decreasing neutralino mass - see the lower boundary of the scatter plot in Fig. 2. Low $m_{\chi}$ configurations are allowed if the primary antiproton excess which they yield does not propagate efficiently from the halo of the Milky Way to the solar system. Thin diffusive halos and strong galactic winds are preferred. This is particularly obvious for a $10 \mathrm{GeV}$ neutralino. Notice how the big (black) circles concentrate in the upper-left corner of the $V_{c}$ versus $L$ diagram. Remark also how the normalization $K_{0}$ of the diffusion coefficient scales with the diffusive halo thickness $L$. In the second panel of Fig. [11] the ratio $K_{0} / L$ lies actually in the range between $10^{-3}$ and $10^{-2} \mathrm{kpc} \mathrm{Myr}^{-1}$ irrespective of $L$. That ratio turns out to be crucial in the diffusion equation. Finally, we do not find any particular correlation between $\delta$ and $L$ even if small values for the latter are still pointed towards in the last panel of Fig. 11]

To conclude our investigation, we would like to assess the potential of low-energy antiproton measurements for discovering heavier neutralinos. For that purpose, we present in the $\chi^{2}$ versus $L$ plane of Fig. 12 the same kind of scatter plot as in panel a of Fig. 7 Neutralino masses of $100 \mathrm{GeV}$ (red stars) and $200 \mathrm{GeV}$ (blue crosses) have been displayed. In the supersymmetric configuration which we have selected for each neutralino mass, the effective annihilation cross-section $\xi^{2}<\sigma_{\text {ann }} v>_{0}$ reaches its maximal value - case A) - so that the antiproton signal is the strongest. At fixed $m_{\chi}$, each star or cross is associated to a combination of galactic propagation parameters yielding a good fit for the $\mathrm{B} / \mathrm{C}$ ratio. Notice how 

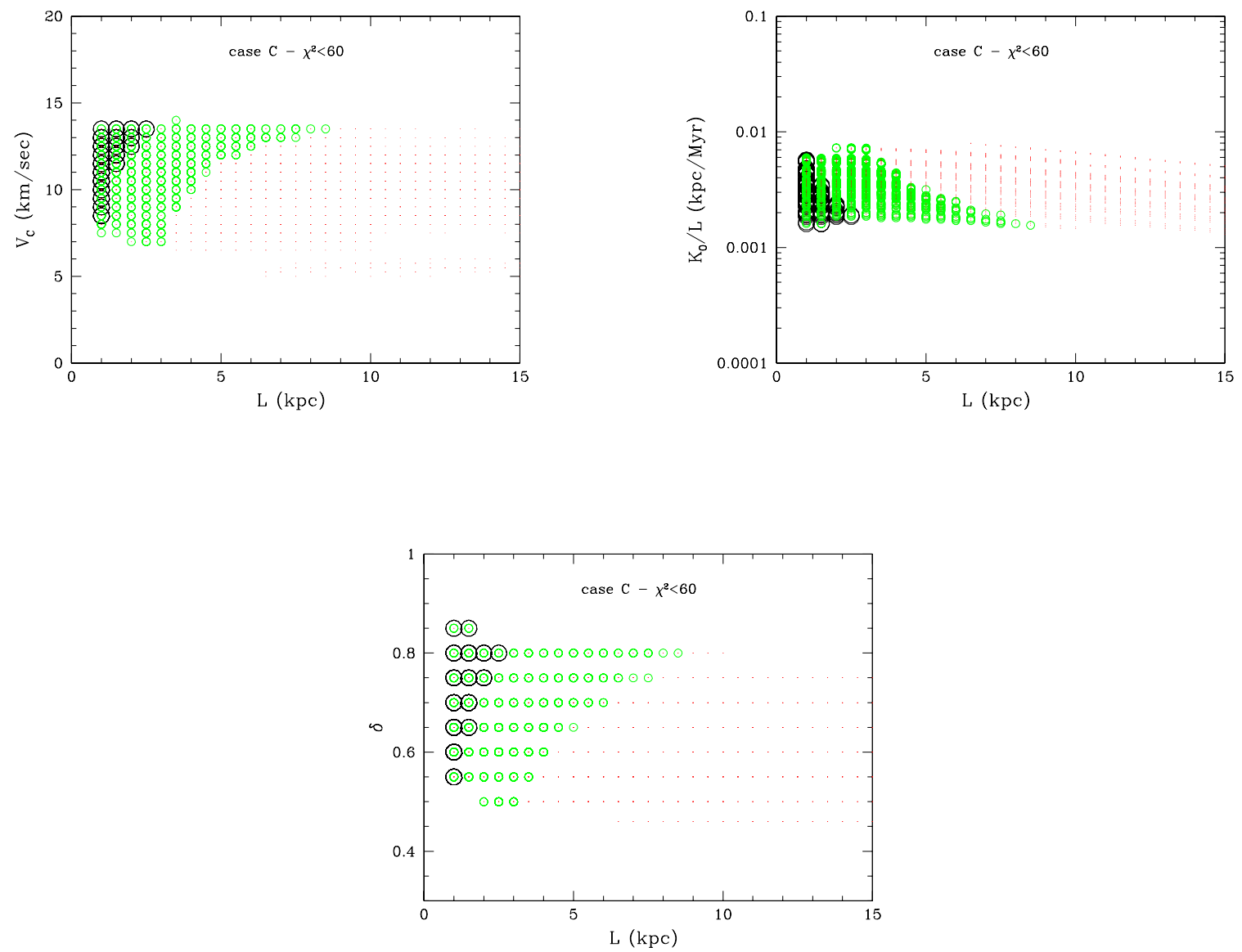

FIG. 11: The three panels feature the same selection of astrophysical configurations from panel $\mathrm{c}$ of Fig. [7 for which the fit to the antiproton data is acceptable with $\chi^{2} \leq 60$. Big black circles, smaller green circles and red dots respectively correspond to a neutralino mass $m_{\chi}$ of 10,20 and $30 \mathrm{GeV}$. The panels successively display the cosmic ray propagation parameters $V_{c}, K_{0} / L$ and $\delta$ as a function of the diffusive halo thickness $L$.

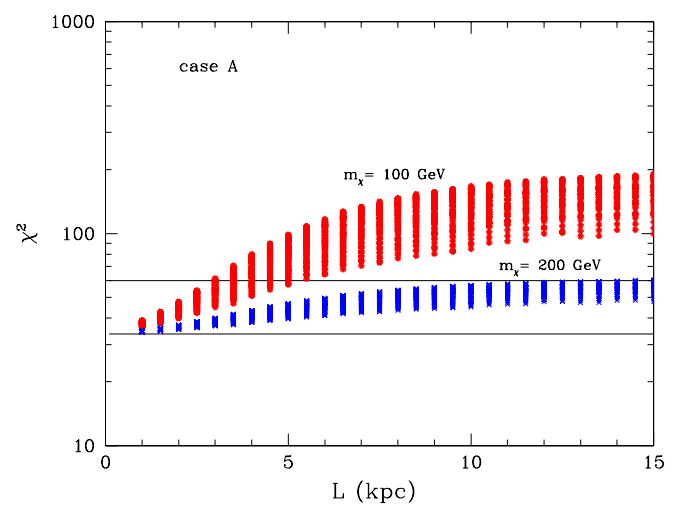

FIG. 12: Same as in Fig. 7 panel a) but for $m_{\chi}=100 \mathrm{GeV}-$ upper branch - and $m_{\chi}=200 \mathrm{GeV}$ - lower branch. Horizontal lines are the same as in Fig. [7]

the constellation of (blue) crosses lies between the two

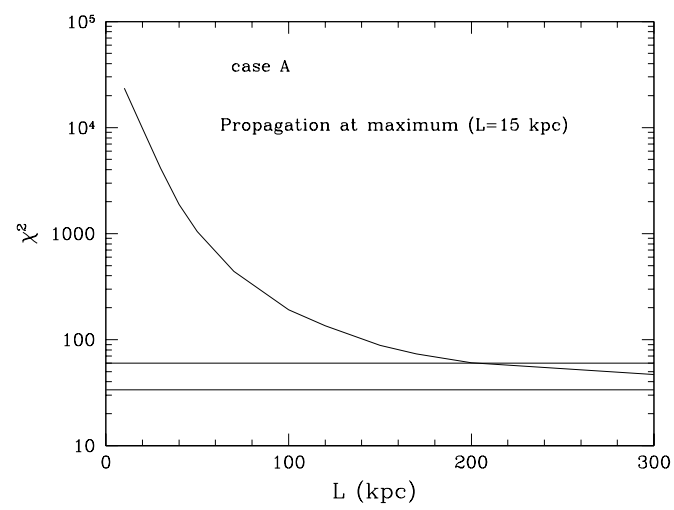

FIG. 13: The $\chi^{2}$ is plotted as a function of the neutralino mass. Case A) has been selected with astrophysical parameters leading to the maximal signal. Horizontal lines are the same as in Fig. [7] 
horizontal lines. We readily conclude that whatever the cosmic ray propagation model is, low-energy antiproton data are useless as long as $m_{\chi} \gtrsim 200 \mathrm{GeV}$. Our statement is supported by Fig. 13] where the maximal possible value of $\chi^{2}$ has been plotted as a function of neutralino mass. The effective annihilation cross-section $\xi^{2}<\sigma_{\text {ann }} v>_{0}$ has been once again tuned to its maximal value - case A) - and the astrophysical configuration max of Tab. \ has been chosen. The $\chi^{2}$ curve drops below the exclusion value of 60 when the neutralino mass exceeds $200 \mathrm{GeV}$. Observations at significantly higher energies will probably be necessary to explore the regime where neutralinos are heavy. We leave such an investigation for a future publication but we cannot resist noticing that the Caprice data above $10 \mathrm{GeV}$ already exhibit an excess with respect to the secondary antiproton background. If such a distortion is confirmed - by the forthcoming Pamela satellite mission [25] for instance - it should have to be explained 11]. Pamela will actually probe the antiproton spectrum between $80 \mathrm{MeV}$ and $190 \mathrm{GeV}$ and collect data during three years starting at the end of 2005. As well as the Pamela experiment, other space missions are planned - such as AMS-02 on board the International Spatial Station 26] or the balloon-borne Bess Polar mission in Antarctica 27] - or in project - such as the GAPS satellite experiment 28]. They will measure the low-energy antiproton spectrum with very high accuracy, and their results will be of great relevance for improving the study developed in the present paper. Improvements in the understanding of the propagation of galactic cosmic rays are foreseeable in the next future, thanks to long and ultra-long duration balloon missions 29] and space-based expriments 26]. Even if the best expectations are deserved to the determination of the diffusion coefficient power spectrum $\delta$, data on the $\mathrm{B} / \mathrm{C}$ quantity in the $\mathrm{GeV} / \mathrm{n}$ region will also constrain the diffusive halo thickness $L$ [30] which, among the astrophysical parameters, plays the most important role in our previous analysis.

Finally, we recall that the conclusions of the present paper are drawn for the case of a smooth distribution of dark matter in the galactic halo. Should the dark matter distribution have some clumpiness, the antiproton signal would be enhanced [31], and consequently some of the constraints on the previously discussed supersymmetric configurations would become more stringent.

\section{Acknowledgments}

We acknowledge Research Grants funded jointly by the Italian Ministero dell'Istruzione, dell'Università e della Ricerca (MIUR), by the University of Torino and by the Istituto Nazionale di Fisica Nucleare (INFN) within the Astroparticle Physics Project. P.S. acknowledges a support from the French Programme National de Cosmologie PNC. F.D. acknowledges support from the A. von Humboldt Stiftung and hospitality from the Max-Planck Institute für Physik in Munich, where part of this work was done.
[1] D.N. Spergel et al., Astrophys. J. Suppl. 148, 175 (2003) astro-ph/0302209.

[2] M. Tegmark et al., Phys. Rev. D 69, 103501 (2004) astro-ph/0310723.

[3] A. Bottino, N. Fornengo and S. Scopel, Phys. Rev. D 67, 063519 (2003) hep-ph/0212379.

[4] A. Bottino, F. Donato, N. Fornengo and S. Scopel, Phys. Rev. D 68, 043506 (2003) hep-ph/0304080

[5] A. Bottino, F. Donato, N. Fornengo and S. Scopel, Phys. Rev. D 69, 037302 (2004) hep-ph/0307303.

[6] A. Bottino, F. Donato, N. Fornengo and S. Scopel, Phys. Rev. D 70, 015005 (2004) hep-ph/0401186.

[7] J. Silk and M. Srednicki, Phys. Rev. Lett. 53, 624 (1984); J. Ellis, R.A. Flores, K. Freese, S. Ritz, D. Seckel and J. Silk, Phys. Lett. B 214, 403 (1988); F. Stecker, S. Rudaz and T. Walsch, Phys. Rev. Lett. 55, 2622 (1985); J.S. Hagelin and G.L. Kane, Nucl. Phys. B 263, 399 (1986); S. Rudaz and F.W. Stecker, Astrophys. J. 325, 16 (1988); F. Stecker and A. Tylka, Astrophys. J. 336, L51 (1989); G. Jungman and M. Kamionkowski, Phys. Rev. D 49, 2316 (1994).
[8] A. Bottino, C. Favero, N. Fornengo, and G. Mignola, Astropart. Phys. 3, 77 (1995).

[9] A. Bottino, F. Donato, N. Fornengo, and P. Salati, Phys. Rev. D 58, 123503 (1998).

[10] L. Bergström, J. Edsjö, and P. Ullio, Astrophys. J. 526, 215 (1999).

[11] F. Donato, N. Fornengo, D. Maurin, P. Salati, R. Taillet Phys. Rev. D 69, 063501 (2004).

[12] T.K. Gaisser, G. Steigman and S. Tilav, Phys. Rev. D 34, 2206 (1986).

[13] A. Bottino, C. Favero, N. Fornengo, G. Mignola and S. Scopel, Proc. of the International Workshop "Doublebeta Decay and Related Topics" (Ed. H.V. KlapdorKleingrothaus and S. Stoica, ECT*/Trento, 1995), 281 (World Scientific, 1996).

[14] A. Bottino, F. Donato, N. Fornengo and S. Scopel, Proc. of "Results and Perspectives in Particle Physics", (Ed. M. Greco, La Thuile, 2001) 135 (INFN Laboratori Nazionali Frascati) hep-ph/0105233.

[15] T. Sjöstrand, P. Eden, C. Friberg, L. Lonnblad, G. Miu, S. Mrenna and E. Norrbin, Comp. Phys. Commun. 135, 
238 (2001).

[16] D. Maurin, F. Donato, R. Taillet, and P. Salati, Astrophys. J. 555, 585 (2001).

[17] D. Maurin, R. Taillet, F. Donato, Astron. \& Astrophys. 394, 1039 (2002).

[18] D. Maurin, R. Taillet, F. Donato, P. Salati, A. Barrau, and G. Boudoul, in research Signposts, "Recent Developments in Astrophysics", astro-ph/0212111.

[19] F. Donato et al., Astrophys. J. 563, 172 (2001).

[20] F. Donato, D. Maurin, and R. Taillet, Astron. \& Astrophys. 381, 539 (2002).

[21] S. Orito, et al. (BESS Collaboration), Phys. Rev. Lett. 84, 1078 (2000).

[22] T. Maeno, et al. (BESS Collaboration), Astropart. Phys. 16, 121 (2001).

[23] M Aguilar, et al. (AMS Collaboration), Phys. Rep. 366,
331 (2002).

[24] M. Boezio, et al. (CAPRICE Collaboration), Astrophys. J. 561, 787 (2001).

[25] http://wizard.roma2.infn.it/pamela/

[26] J. Burger, Eur. Phys. J. C 33, s941 (2004).

[27] S. Haino et al., Nucl. Instrum. Meth. A 518, 167 (2004).

[28] K. Mori et al., Astrophys. J. 566, 604 (2002).

[29] E.S. Seo et al., Adv. in Space Res. 33, 1777 (2004).

[30] A. Castellina and F. Donato, Astropart. Phys., in press, astro-ph/0504149.

[31] H. Bengtsson, P. Salati and J. Silk, Nucl. Phys. B 346, 129 (1990); J. Silk and A. Stebbins, Astrophys. J. 411, 439 (1993); V. Berezinsky, A. Bottino and G. Mignola, Phys. Lett. B 391, 355 (1997). 